\title{
Opportunities and Challenges of Data Migration in Cloud
}

\author{
Ruhul Amin ${ }^{1}$, Siddhartha Vadlamudi ${ }^{2 *}$, Md. Mahbubur Rahaman ${ }^{3}$ \\ ${ }^{1}$ Senior Data Entry Control Operator (IT), ED-Maintenance Office, Bangladesh Bank (Head Office), \\ Dhaka, BANGLADESH \\ ${ }^{2}$ Software Engineer II, Xandr, AT\&T Services Inc., New York, USA \\ ${ }^{3}$ Assistant Professor, Department of Business Administration, Leading University, Sylhet, BANGLADESH \\ *Corresponding Contact: \\ Email: vadlamudisiddhartha@gmail.com
}

\begin{abstract}
Cloud data migration is the process of moving data, localhost applications, services, and data to the distributed cloud processing framework. The success of this data migration measure is relying upon a few viewpoints like planning and impact analysis of existing enterprise systems. Quite possibly the most widely recognized process is moving locally stored data in a public cloud computing environment. Cloud migration comes along with both challenges and advantages, so there are different academic research and technical applications on data migration to the cloud that will be discussed throughout this paper. By breaking down the research achievement and application status, we divide the existing migration techniques into three strategies as indicated by the cloud service models essentially. Various processes should be considered for different migration techniques, and various tasks will be included accordingly. The similarities and differences between the migration strategies are examined, and the challenges and future work about data migration to the cloud are proposed. This paper, through a research survey, recognizes the key benefits and challenges of migrating data into the cloud. There are different cloud migration procedures and models recommended to assess the presentation, identifying security requirements, choosing a cloud provider, calculating the expense, and making any essential organizational changes. The results of this research paper can give a roadmap for data migration and can help decision-makers towards a secure and productive migration to a cloud computing environment.
\end{abstract}

Key words:

Data migration, cloud computing, cloud, enterprise systems 


\section{INTRODUCTION}

Cloud computing platforms such as those offered by Amazon Web Services, ATT, Intel, IBM, and Google cloud have gotten critical consideration in addressing the necessities of organizations i.e. computational force, decreasing the expense of infrastructure and maintenance, and proficient resource allocation (Vadlamudi, 2015; Buyya et al., 2008; Kocak et al., 2013). These advantages are acknowledged by a wide scope of services which are generally open, progressively acquirable, and releasable, and through usage-based costing models. Cloud computing is an essential shift in delivering IT services with an expansive effect on organizations. Cloud computing of existing systems, as a backbone of enterprises, has huge implications for organizational performance.

The main issue while adopting the cloud-based platforms is the migration of data into the cloud. There are different ways and types of services the cloud framework offers. With this current innovation, a significant part of the challenges is needed to be resolved. One significant challenge to the adap-tion of cloud computing has been the absence of visibility into the security of the platform (Donepudi et al., 2020b). Cloud service providers are approached to ensure and constantly check the viability of their security methodologies (Rahman et al., 2020). While choosing a cloud provider, security must be a requirement and a top priority. Organizations need to ensure that their supplier can uphold their requirements as far as security and compliance (Vadlamudi, 2017).

Organizations can moderate the risks of cloud computing by developing a comprehensive risk assessment before choosing a supplier and launching a service. Data integrity is likewise a challenge for data migration into the cloud. If your data isn't sensitive, you just have the only issue of ensuring it gets into the cloud seamlessly. This is a problematic challenge you can address by migrating your data with a tool that checks the integrity of data.

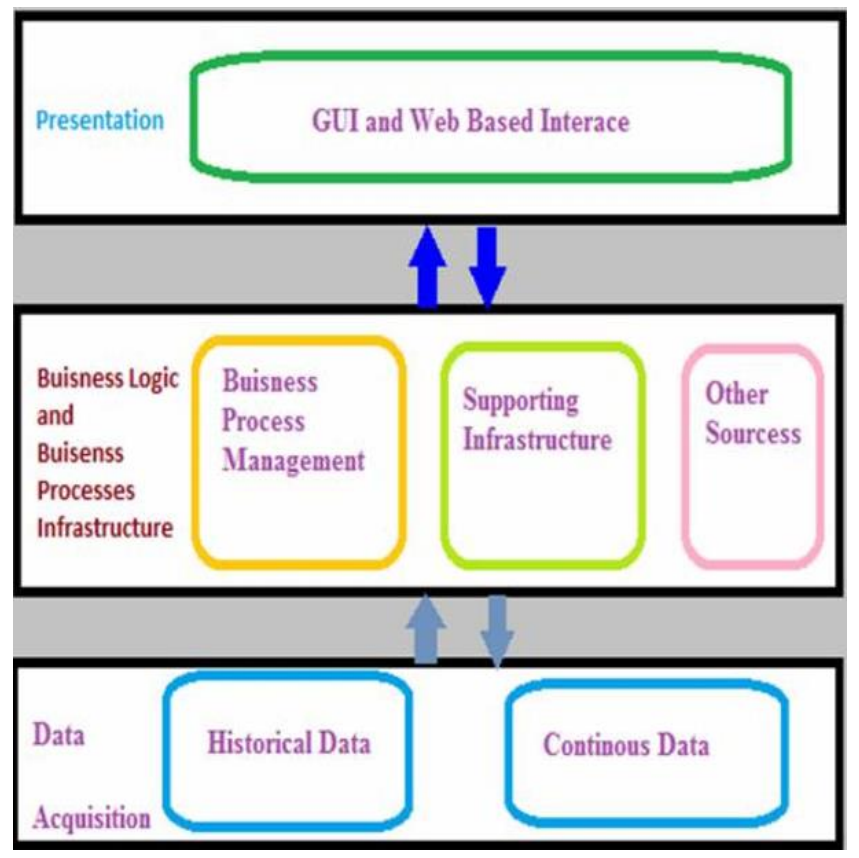

Figure 1: Data Migration in Cloud Layers 
In this paper, we review the research achievements related to cloud migration, do an extensive analysis of existing migration techniques, and characterize them into three migration strategies. The main purpose of this paper is to introduce the opportunities and advantages of data migration into the cloud as well as featuring the challenges of the migration. Moreover, focusing on how cloud migration procedures and models can assist with eliminating these challenges.

\section{LITERATURE REVIEW}

Analyzing cloud migration as an instance of shifting from client-based frameworks to cloudbased frameworks is an on-going research topic and has been studied in the literature from a variety of including models for organizations to decide on the sustainability of cloud migration (Misra and Mondal, 2011). The research presents and approves a model showing pull factors (for example, failures with customer IT and relative usability) and push factors (for example learning cost, setup cost, and security measures). In any case, the authors express that regardless of the presence of these elements, users may not completely migrate to the cloud because of challenges like shifting costs and personal judgment. What's more, in (Pallas, 2014), the office hypothesis has been utilized to comprehend and analyze the irreconcilable circumstances happening between cloud providers and buyers. The research sorts and evaluates countermeasures to these challenges and identifies possible specialized and non-specialized ways to broaden the sustainability of cloud migration. Using the exchange cost hypothesis, another research (Yigitbasioglu, 2014), observed that organizations have concerns about the cloud services since liabilities may not be indicated as traditional approaches do and the idea of cloud services includes various jobs and new abilities. Such vulnerability shows up as an advantage of cloud service providers, classification, integrity, and accessibility of data. The above research observes cloud migration as a 'black box' by not narrowing its core interest down on key operational challenges engaged with the migration cycle.

Moreover, our examination is a response to experimentally explore how the migration process of data to the cloud is conducted; and subsequently, the challenges and possible countermeasures on this progress are provided for both the researchers and professionals as a guide.

\section{Migration Strategies in Cloud-Based Architecture}

Through comparing and analyzing, we can categorize the migration into three strategies vitally: migration to IaaS, migration to PaaS, and migration to SaaS. The primary technique carries out migration exclusively by transferring the whole application/framework to the cloud by utilizing IaaS. The framework will be moved to the cloud by refactoring as shown by the foundation of PaaS in the next technique. As to the migration to SaaS, it tends to be divided into three sub-techniques, namely replacing by SaaS, revising based on SaaS, and reengineering to SaaS.

To the main sub-strategy, systems will be replaced by business programming delivered as a cloud service. As to the second sub-strategy, some usefulness of systems will be replaced by cloud services. To the third sub-strategy, systems will be reengineered to cloud services. Organizations frequently move their systems to cloud platforms by adopting the first methodology. 
To this methodology, the migration is moderately simple to carry out and has great costsaving advantages. Be that as it may, the migration couldn't take full advantage of the cloud platform in this case. To the second strategy, systems should be adapted according to the targeted platform, which can bring disadvantages including missing features, transitive risk, and framework lock-in. To the SaaS-related strategy, if the system is replaced by commercial software delivered as a service, the migration effort will be reduced incredibly and reengineering is pointless. When replacing some business logic with existing cloud service, the adaption to the system is essential. Yet, to re-engineer the whole system to the cloud, the related work will be challenging and may require reverse engineering, structure upgrade, service generation, etc.

The proposed procedures acknowledge classification to cloud migration basically, which can cover all migration cases. Through these migration strategies, the cloud migration process can be better perceived and evaluated.

Table 1: Required Measures and Features for Data Migration in the Cloud

\begin{tabular}{|l|l|}
\hline Measures & Required Features \\
\hline High Performance & $\begin{array}{l}\text { Maximize throughput using procedures, for example, pipelining and } \\
\text { equal data streams. }\end{array}$ \\
\hline Reliable & $\begin{array}{l}\text { At each phase of data transfer, checks shift performance, recovers } \\
\text { from mistakes by retrying moves, and advises clients of different events } \\
\text { (like risks and success). }\end{array}$ \\
\hline Secure & $\begin{array}{l}\text { Implementation of safety approaches for user authentication and, } \\
\text { approval safely deals with the capacity and transmission of credentials } \\
\text { to endpoints for verification, and supports data encryption. }\end{array}$ \\
\hline $\begin{array}{l}\text { Third-party } \\
\text { Transfer }\end{array}$ & $\begin{array}{l}\text { Third-party transfers between two remote endpoints. That is, } \\
\text { instead of keeping a persistent connection with an endpoint, clients } \\
\text { can begin a transfer and afterward let the Centralized framework } \\
\text { monitor it during the transfer. }\end{array}$ \\
\hline High accessibility & $\begin{array}{l}\text { Dispersed, Replicated, and redundant facilitating model need to be } \\
\text { deployed for high accessibility of information. }\end{array}$ \\
\hline Available & $\begin{array}{l}\text { In a software-as a-service (SaaS) provider, users can access its } \\
\text { functionalities without installing client software on a local } \\
\text { environment. }\end{array}$ \\
\hline $\begin{array}{l}\text { On-demand self } \\
\text { service }\end{array}$ & $\begin{array}{l}\text { Enabling a Big Data cloud service customer to self-provision cloud } \\
\text { service- both physical and virtual - automatically and with minimal } \\
\text { communication including the cloud service providers. }\end{array}$ \\
\hline $\begin{array}{l}\text { Quick flexibility } \\
\text { and scalability }\end{array}$ & $\begin{array}{l}\text { This empowers Big Data cloud resources to be quick, flexibly, and } \\
\text { naturally scaled out up, out, and down on demand. }\end{array}$ \\
\hline
\end{tabular}

\section{Migration to IaaS}

Infrastructure as a service is a type of hosting, which incorporates network access, routing services, and storage. IaaS supplier generally provides hardware and authoritative services used to store applications and a platform for running applications. A virtual machine is built for an application, which is stacked with all the products that will at last run the cloud. When the virtual machine is transferred to IaaS vendors hosting environment and deployed to run. IaaS is the most ideal migration strategy for moving applications to the cloud when there is no opportunity to reengineer the applications for a cloud (Bhardwaj et al., 2010). 
Other than the technology domain that should be considered, there are various components to weigh before relocating the whole system to IaaS like significant prerequisites, financial conditions, and culture shift, etc. If these conditions can't be fulfilled, migration of the framework to IaaS will be harder (Donepudi et al., 2020a).

In addition to the advantages of utilizing the cloud, huge risks, for example, decay of customer support and service quality, expanded dependence on the third-party stack, absence of supporting sources, etc. will come up. Project managers need to consider the overall administrative ramifications of the progressions brought by moving the system to the cloud, so they can try not to implement local optimizations at the cost of organizationwide performance.

So, clients have full advantages on the allocated virtual machine (VM) in migration to IaaS. They could do anything to the VM, yet there are a few elements, for example, dynamic resource management and amount of data stream, that should be considered before lead migration.

\section{Migration to PaaS}

Platform as a service is an application development and deployment platform delivered as a service to developers over the internet, which gives the hardware in addition to a specific amount of applications like databases, middleware, and development tools. Migration dependent on PaaS isn't compulsory for resource management, but it is needed to make the system viable with the prerequisite of the PaaS vendor.

Some of the rules are confined to the PaaS and not pertinent to other general cases. For the general cases, further checking steps are essential for application migration to PaaS, including programming language, database, limitations, and impediments of the chosen PaaS other than checking specific needs identified with hardware, programming, and input data as discussed in migration to IaaS. The scope of migration software system for the cloud is identified within all exercises in migration that start from getting familiar with the application, the targeted cloud platform, and the third-party device, at that point to build the environment and prepare for migration, as well as to change and test to ensure that the application properly runs in the cloud.

In a nutshell, PaaS supplies a total cloud IT stack for software development and delivery, which makes it possible to build "viable" cloud applications and deliver them in an adaptable and versatile environment. Additionally, it likewise creates various limitations at each innovation layer of the application stack as follows:

- Programming language. Database.

- Middleware.

- Third-party library.

- Restriction of the selected PaaS platform.

\section{Migration to SaaS}

To provide some direction on modifying the design of a service-based framework for cloud computing, past studies revealed a few experiences when they undertook a research project pointed towards modernizing an open-source software framework for utilizing the adaptability and versatility of the cloud computing paradigm. During the time spent migration, the key prerequisites that can be relocated to the cloud are identified first. Having 
identified the key requirements, the next step is to break down those prerequisites with regards to the current engineering to acquire a decent understanding of the types of architectural changes that should have been made. At long last, the design will be modified as per the identified engineering modification. Since this work focused on the migration of the service-based framework to a cloud computing platform, it didn't include reverse engineering.

Along with the migration strategy, some research on the performance of migration to SaaS was done. Current methodologies are frequently restricted to some specific cloud conditions or don't provide digital support to be aligned with a cloud environment. CloudMig, a model-based methodology, focuses on the SaaS provider point of view and works with the migration of big business software frameworks towards generic IaaS and PaaS-based cloud environments. CloudMIG can produce extensive parts of resource-proficient objective engineering using rule-based heuristics, so it helps SaaS providers to semi-automatically migrate existing applications to the cloud computing platform.

In conclusion, migration to SaaS needs to consider the particular migration technique as per the framework or application and existing SaaS. On the off chance that current SaaS has a similar business application of the framework, users can replace that framework with SaaS. When some business functionality has been modernized by existing SaaS, the frame-work can be modernized by reexamining the whole application dependent on existing SaaS. Users can take great advantage of the virtue of the cloud by reengineering the existing framework to SaaS, yet the challenge can't be overlooked.

\section{Opportunities Advantages of Data Migration in Cloud}

\section{Adaptable IT resources}

By scalable IT resources, most cloud service providers will allow an organization to increase their current resources to complement their business needs or changes. A few users may require a fast change in terms of IT resources, and this will empower to help business development without costly hardware to support the existing framework infrastructure. Resource management can be taken care of through cloud recourses effectively if any application is having expanded traffic, while it's not a simple task to increase demand on the resources through conventional computing techniques and environments (Vadlamudi, 2016).

\section{Cost-saving solution}

Cloud computing is a highly emerging innovation due to its features like scalability, reliability, and exceptionally accessible model for enterprises. Data migration to the cloud is a cost-saving solution as it is compared with on-premises costs like equipment, programming, support, time investment, employees, and depreciation cost. Cost is one of the vital advantages for organizations, so they can focus on their core business objectives while putting their primary infrastructure services on the cloud computing platforms and have them working from there.

Then again, cloud computing is a greater environment compared with on-premises frameworks, saves energy, and provides the cloud's intrinsic highlights reducing the number of physical materials (Bedward \& Fokum, 2014). 


\section{Security features}

Data is imperative for any organization, and cloud providers should think about the current realities of integrity and re-liability of basic data, which is fundamental in the present business scene. Cloud provider's commitment ensures that their infrastructure is secure, and their customer's data and applications are all around ensured. Cloud service providers give an undeniable level of security convention to check data assurance by using high-end encryption systems. The complex data centers of cloud providers are based on layered security approaches which incorporate data encryption, password management, solid access controls, and compliance with generic security audits (Ahmed et al., 2020).

\section{Opportunity to manage incomings and outgoings}

Introducing cloud infrastructure solutions introduces itself as a chance to improve the management of income and outgoings for both account staff and users. Cloud infrastructure facilitates the management of income for finance staff as the cloud model has a negligible upfront cost and monthly cost, and it additionally limits variability of use on electricity. These are an advantage, as opposed to an in-house data center, as upfront expenses of purchasing equipment are high and customers can be delayed to pay, bringing about income challenges.

Cloud infrastructure solutions likewise present numerous opportunities for managing income for customers, sales, and marketing staff, as new models can be offered to them. This is an advantage, as opposed to in-house data centers which require a pricing model including an enormous upfront cost in addition to monthly costs (because of income issues), as customers can be offered more choice over how they need to pay or on the other hand the finance office can decide to get the infrastructure outsourcer to charge their customers reducing the finance departments' administrative burden.

\section{Opportunity to develop new skillsets}

Cloud infrastructure platforms present a chance for support managers, developers, sales and marketing staff to develop new skills. For support staff and developers, it is a chance to develop new skills in cloud computing. This is an advantage as the support staff and developers will grow their current involvement in information on dealing with an innovation that will be in demand all through the IT business for quite a long time to come. For sales and marketing staff it presents a chance to develop new skills in product/service creation and launching. This is an advantage to sales and marketing staff as it will grow their current abilities and experience empowering their career progression.

\section{Opportunity for organizational development}

Cloud infrastructure solutions present an opportunity for sales and marketing staff to create new product/service contributions that may engage a bigger piece of the pie because of cloud-infrastructures properties of scalability and it is cost-saving as opposed to an in-house data center. This is an advantage as it might work with sales staff targets by allowing them to target market segments earlier not attracted by limitations of scalability.

\section{Challenges Risks of Data Migration in Cloud}

\section{Choosing the right cloud vendor}

Data management and data migration are fundamental re-search challenges, and it is never pretty much as basic as migrating data from conventional platforms to the cloud. Even after 
the SWOT (Strength, Weakness, Opportunities, and Threats) analysis, it isn't smooth for an organization to choose an appropriate cloud vendor. In the cloud market driving key players, Google Cloud Platform (GCP), Amazon Web Services (AWS), and Microsoft Azure are persistently looking for ways to deal with separating themselves from competitors. Accordingly, organizations must ask cloud vendors if they have appropriate data migration tools to move data by considering the elements of vendor lock-in and portability (the ability of software to be moved from one machine or framework to the next).

\section{Trust deficit about cloud security}

Even though the cloud market has been advancing its most recent information security model, risks surrounding information security make uncertainty and rethink on storing all the basic and secret information in the cloud. This trust deficit impacts all significant stakeholders like individual citizens, organizations, and governments. As information stored in the cloud is effectively accessible from any place, data breaches because of low security or hacking can bring about a tradeoff of individual and business information. The organizations hosted their information locally with having full control and authority. When they decide to migrate to the cloud, they may feel more powerless because hackers will in general target huge data centers.

\section{Departmental downsizing}

Cloud framework implementation presents a risk of scaling back to IT support departments. IT support departments are at risk of downsizing if most of their work involves hardware and organization support. This is a risk since cloud suppliers will be liable for keeping up these parts of help making the capability unnecessary inside the IT support department. Both IT support managers and IT support engineers will be affected as support engineers may lose their jobs and the IT support managers may lose impact as they have a little department to manage.

\section{Lack of supplementary resources}

Cloud framework implementation presents a risk of resource scarcity in IT support and sales/marketing departments. There is a risk of briefly upsizing the IT support departments to adapt to migration and furthermore the general lack of data and experience held my support engineers with respect to cloud frameworks. This is a risk because staff may at first require more opportunity to perform something very similar assignments due to having to learn how to do as such in the cloud environment. There is a risk of briefly upsizing sales/marketing to adapt to the creation and launching of new cloud-based products/services. This is a risk because sales and marketing staff should create appropriate methodologies and materials to ensure the marketplace knows about the product/service offering.

\section{CONCLUSION}

Cloud computing is a disruptive technology that is set to change how IT systems are deployed as a result of its obviously modest, simple and versatile nature. The discoveries of this contextual research show that cloud computing can be a fundamentally less expensive option as compared to buying and maintaining framework infrastructure in-house. Moreover, cloud computing might actually eliminate many support-related issues since there would be no actual infrastructure to keep up. Despite these benefits, this contextual research showed that some significant challenges and risks should be considered before organizations could migrate their IT frameworks to the cloud. 
By exploring the current literature, we classified the data migration into three strategies, then audited and analyzed every migration strategy. Also, related development tools are surveyed. In light of the current analysis and application status, some future work is recognized including holistic system, overhaul, and adaption to application for special migration, design refactoring, integrated development environment, etc.

To appropriately execute the conclusion and ideas discussed in this paper, some extra work must be done. The future of cloud computing should be in discussion with open source and closed source cloud environment and their solution of security approaches. Cybersecurity is an intriguing topic in all organizations nowadays, and it will stay critical in terms of cloud security standards adopted by cloud vendors.

\section{REFERENCES}

Ahmed, A. A. A., Donepudi, P. K., \& Asadullah, A. B. M. (2020). Artificial Intelligence in Clinical Genomics and Healthcare. European Journal of Molecular \& Clinical Medicine, 7(11), 1194-1202, https: / / ejmcm.com/? action=article\&au $=24014$

Bedward, R., \& Fokum, D. T. (2014). A Cloud computing adoption approach for Jamaican institutions. IEEE SOUTHEASTCON 2014, p. 1-6. https://doi.org/10.1109/SECON.2014.6950693

Bhardwaj, S.; Jain, L.; \& Jain, S. (2010). Cloud computing: A study of infrastructure as a service (IAAS). International Journal of Engineering and Information Technology, 2(1), 60-63.

Buyya, R.; Yeo, C. S.; and Venugopal, S. (2008). Market-oriented cloud computing: Vision, hype, and reality for delivering it services as computing utilities. in High Performance Computing and Communications, HPCC'08. 10th IEEE International Conference, pp. 5-13.

Donepudi, P. K., Ahmed, A. A. A., Hossain, M. A., \& Maria, P. (2020a). Perceptions of RAIA Introduction by Employees on Employability and Work Satisfaction in the Modern Agriculture Sector. International Journal of Modern Agriculture, 9(4), 486-497. https://doi.org/10.5281/zenodo.4428205

Donepudi, P. K., Ahmed, A. A. A., Saha, S. (2020b). Emerging Market Economy (EME) and Artificial Intelligence (AI): Consequences for the Future of Jobs. Palarch's Journal of Archaeology of Egypt/Egyptology, 17(6),

17(6),

5562-5574. https://archives.palarch.nl/index.php/jae/article/view/1829

Kocak, S. A.; Miranskyy, A.; Alptekin, G. I.; Bener, A. B.; and Cialini, E. (2013). The Impact of Improving Software Functionality on Environmental Sustainability. on Information and Communication Technologies, p. 95.

Misra, S. C., and Mondal, A. (2011). Identification of a company's suitability for the adoption of cloud computing and modeling its corresponding Return on Investment. Mathematical and Computer Modelling, 53, 504-521.

Pallas, F. (2014). An agency perspective to cloud computing. in International Conference on Grid Economics and Business Models, pp. 36-51.

Rahman, M. M., Chowdhury, M. R. H. K., Islam, M. A., Tohfa, M. U., Kader, M. A. L., Ahmed, A. A. A., \& Donepudi, P. K. (2020). Relationship between Socio-Demographic Characteristics and Job Satisfaction: Evidence from Private Bank Employees. American Journal of Trade and Policy, 7(2), 6572. https://doi.org/10.18034/ajtp.v7i2.492

Vadlamudi, S. (2015). Enabling Trustworthiness in Artificial Intelligence - A Detailed Discussion. Engineering International, 3(2), 105-114. https://doi.org/10.18034/ei.v3i2.519

Vadlamudi, S. (2016). What Impact does Internet of Things have on Project Management in Project based Firms?. Asian Business Review, 6(3), 179-186. https://doi.org/10.18034/abr.v6i3.520 
Vadlamudi, S. (2017). Stock Market Prediction using Machine Learning: A Systematic Literature Review. American Journal of Trade and Policy, 4(3), 123-128. https://doi.org/10.18034/aitp.v4i3.521

Yigitbasioglu, O. (2014). Modelling the intention to adopt cloud computing services: a transaction cost theory perspective. Australasian Journal of Information Systems, vol. 18.

$--0--$

\section{How to Cite:}

Amin, R., Vadlamudi, S., \& Rahaman, M. M. (2021). Opportunities and Challenges of Data Migration in Cloud. Engineering International, 9(1), 41-50. https://doi.org/10.18034/ei.v9i1.529 